\title{
Innovación e Inteligencia Artificial en Medicina
}

\author{
Dr. Carlos Ingino, ENERI, Buenos Aires, Argentina \\ Email: info@diagnosticojournal.com \\ Diagnostico Journal
}

Octubre 2019

\begin{abstract}
Diagnostico Journal (Oct. 2019; https://diagnosticojournal.com/articulos/792/innovacion-einteligencia-artificial-en-medicina) -- La inteligencia artificial (IA) ha sido una innovación disruptiva en el mundo de la salud y la medicina. Además del área de la investigación, la IA puede otorgar soluciones algorítmicas en cuestiones clínicas para ayudar al diagnóstico, pronóstico y tratamiento de los pacientes, así como en el reconocimiento por software de patrones visuales en el campo de la radiología y la interpretación de imágenes.

De acuerdo a un estudio biblio-métrico recientemente publicado por Tran y col, el número de investigaciones en aplicaciones de IA en medicina se ha triplicado en los últimos 3 años, con la enfermedad cardíaca y el stroke como dos de los tres tópicos de mayor interés. El potencial de la IA para ayudar en la toma de decisiones clínicas y el manejo del stroke y enfermedades cardíacas es múltiple y está en constante expansión (Figura 1). El desarrollo de la investigación y las publicaciones de las mismas ha tenido especial crecimiento en USA (44.1\% de las publicaciones mundiales y el resto dividido en más de 15 países).1
\end{abstract}

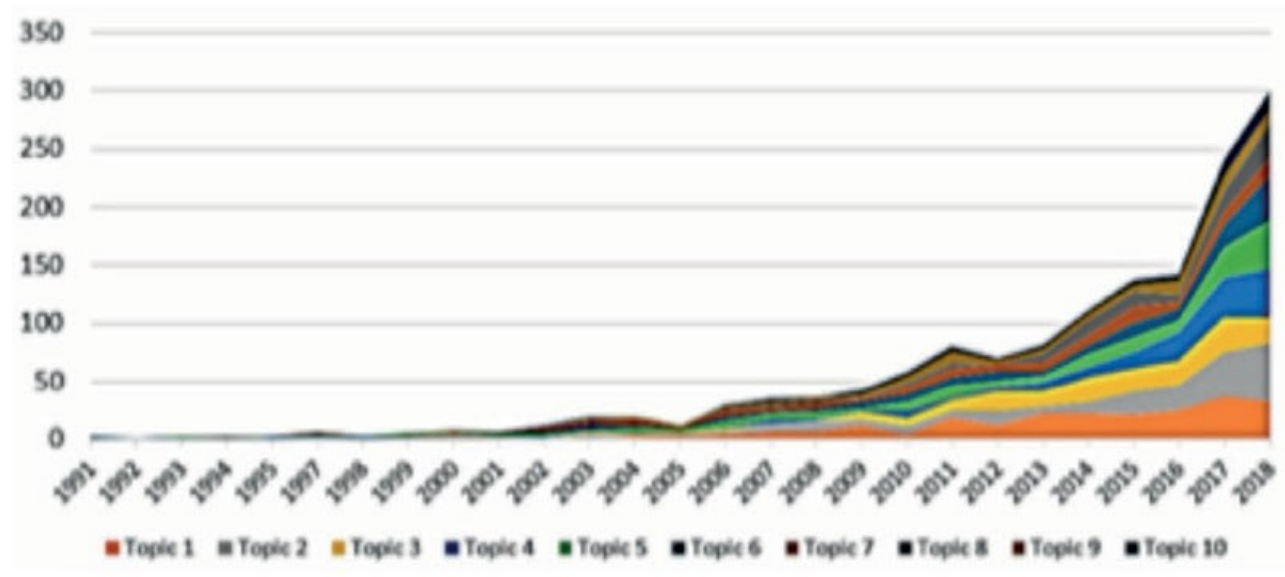

Figura 1. Cambios en las aplicaciones de la investigación de la IA en stroke y enfermedad cardíaca durante 1991-2018.1 
A modo de ejemplo, podrían mencionarse algunas aplicaciones de "machine learning" (aprendizaje automático) vinculadas a nuestra área de máxima innovación, como son:

a) predictores de placas coronarias de alto riesgo o ausencia de coronariopatía mediante bio-marcadores en paciente con sospecha de coronariopatía; 2

b) selección de pacientes añosos apropiados para tratamiento endovascular para reducir riesgo de sangrado luego de la trombectomía mecánica;3

c) severidad de la estenosis coronaria y de la magnitud de la isquemia;4,7

d) predicción de la extensión de la secuela luego del stroke.8,12

Mediante el aporte de las bases de datos masivas, de múltiples parámetros y de resultados clínicos, el aprendizaje automático es muy útil para tamizar los datos y detectar patrones que ayudan al diagnóstico, por ejemplo, en pacientes con angina de pecho mediante anotaciones clínicas;13 a predecir la mortalidad de la hemorragia cerebral;14 o a identificar pacientes con insuficiencia cardíaca de los datos de registros electrónicos médicos.15

Otra área de impactante desarrollo es el despegue de la IA robótica.1,16 A medida que la tecnología robótica se traslade al campo de la salud, los beneficios generados impactarán profundamente en diferentes áreas, como es el caso, por ejemplo, de las neuro-prótesis, la rehabilitación psicofísca robótica, o las herramientas robóticas para diferentes tipos de cirugía.1,16

La IA se enfrenta a algunos desafíos que trascienden a las especialidades médicas. El manejo de los datos, su utilidad clínica y la confi abilidad de los mismos son de relevante importancia.16,18 El manejo de los datos debe sustentarse en un modelo de recopilación validado, funcionalmente apto y con adecuado resguardo de la confi dencialidad. Asimismo, las enfermedades deben contar con grandes bases de datos para su caracterización, que a la vez carezcan de desviaciones.

\section{Referencias}

1. Tran, BX, Vu GT, Ha GH, et al. Global Evolution of Research in Artificial Intelligence in Health and Medicine: A Bibliometric Study. J Clin Med. 2019;8:360.

2. Bom MJ, Levin E, Driessen RS, et al. Predictive value of targeted proteomics for coronary plaque morphology in patients with suspected coronary artery disease. EBioMedicine. 2019;39:109-117

3. Alawieh A, Zaraket F, Alawieh MB, et al. Using machine learning to optimize selection of elderly patients for endovascular thrombectomy. J Neurolnterv Surg. 2019;11:847851

4. Wan $\mathrm{T}$, Feng $\mathrm{H}$, Tong $\mathrm{C}$, et al. Automated identification and grading of coronary artery stenosis with X-ray angiography. Comput Methods Programs Biomed. 2018;167:1322.

5. Van Hamersvelt RW, Zreik M, Voskuil M, et al. Deep learning analysis of left ventricular myocardium in CT angiographic intermediate-degree coronary stenosis improves the 
diagnostic accuracy for identification of functionally significant stenosis. Eur Radiol. 2019;29:2350-2359

6. Von Knebel Doeberitz PL, De Cecco CN, Schoepf UJ, et al. Coronary CT angiographyderived plaque quantification with artificial intelligence $\mathrm{CT}$ fractional flow reserve for the identification of lesion-specific ischemia. Eur Radiol. 2019; 29:2378-2387.7. Hae H, Kang SJ, Kim WJ, et al. Machine learning assessment of myocardial ischemia using angiography: Development and retrospective validation. PLoS Med. 2018;15:e1002693

8. Heo J, Yoon JG, Park H, et al. Machine Learning-Based Model for Prediction of Outcomes in Acute Stroke. Stroke. 2019;50:1263-1265

9. Pinto A, McKinley R, Alves V, et al. Stroke Lesion Outcome Prediction Based on MRI Imaging Combined with Clinical Information. Front Neurol. 2018;9:1060

10. Kemmling A, Flottmann F, Forkert ND et al. Multivariate dynamic prediction of ischemic infarction and tissue salvage as a function of time and degree of recanalization. $\mathrm{Br} \mathrm{J}$ Pharmacol. 2015;35:1397-1405.

11. McKinley, R.; Hani, L.; Gralla, J.; El-Koussy, M.; Bauer, S.; Arnold, M.; Fischer, U.; Jung, S.; Mattmann, K.; Reyes, M.; et al. Fully automated stroke tissue estimation using random forest classifiers (FASTER). J. Cereb. Blood Flow Metab. 2017, 37, 27282741.

12. Lucas, C.; Kemmling, A.; Bouteldja, N.; Aulmann, L.F.; Mamlouk, A.M.; Heinrich, M.P. Learning to Predict Ischemic Stroke Growth on Acute CT Perfusion Data by Interpolating Low-Dimensional Shape Representations. Front. Neurol. 2018, 9, 989

13. Pakhomov SS, Hemingway $\mathrm{H}$, Weston SA, et al. Epidemiology of angina pectoris: Role of natural language processing of the medical record. Am Heart J. 2007;153:666-673

14. Peng SY, Chuang YC, Kang TW, et al. Random forest can predict 30-day mortality of spontaneous intracerebral hemorrhage with remarkable discrimination. Eur J Neurol. 2010;17:945-950

15. Patel YR, Robbins JM, Kurgansky KE, et al. Development and validation of a heart failure with preserved ejection fraction cohort using electronic medical records. BMC Cardiovasc. Disord. 2018;18:128

16. Loh $\mathrm{E}$. Medicine and the rise of the robots: A qualitative review of recent advances of artificial intelligence in health. BMJ Lead. 2018;2:59-63

17. Char DS, Shah NH, Magnus D. Implementing Machine Learning in Health CareAddressing Ethical Challenges. N Engl J Med. 2018;378:981-983.

18. Panch T, Szolovits $P$, Atun R. Artificial intelligence, machine learning and health systems. J Glob Health. 2018;8:020303

19. Deo RC. Machine Learning in Medicine. Circulation 2015;132:1920-1930. 